\title{
Guidance for Using Tafenoquine for Prevention and Antirelapse Therapy for Malaria — United States, 2019
}

\author{
Julia C. Haston, $\mathrm{MD}^{1}$; Jimee Hwang, $\mathrm{MD}^{2,3}$; Kathrine R. Tan, $\mathrm{MD}^{2}$
}

An estimated 219 million cases of malaria occurred worldwide in 2017, causing approximately 435,000 deaths (1). Malaria is caused by intraerythrocytic protozoa of the genus Plasmodium transmitted to humans through the bite of an infective Anopheles mosquito. Five Plasmodium species that regularly cause illness in humans are $P$. falciparum, P. vivax, $P$. ovale, $P$. malariae, and P. knowlesi (2). The parasite first develops in the liver before infecting red blood cells. Travelers to areas with endemic malaria can prevent malaria by taking chemoprophylaxis. However, most antimalarials do not kill the liver stages of the parasite, including hypnozoites that cause relapses of disease caused by $P$. vivax or P. ovale. Therefore, patients with these relapsing species must be treated with two medications: one for the acute infection, and another to treat the hypnozoites (antirelapse therapy). Until recently, primaquine was the only drug available worldwide to kill hypnozoites. Tafenoquine, a long-acting 8 -aminoquinoline drug related to primaquine, was approved by the Food and Drug Administration (FDA) on July 20, 2018, for antirelapse therapy (Krintafel) and August 8, 2018, for chemoprophylaxis (Arakoda) $(3,4)$. This report reviews evidence for the efficacy and safety of tafenoquine and provides CDC guidance for clinicians who prescribe chemoprophylaxis for travelers to areas with endemic malaria and treat malaria.

\section{Background}

In 2016, a total of 2,078 imported malaria cases were reported in the United States; of the 1,853 (89.2\%) cases with known species, $76.6 \%$ were caused by $P$. falciparum, $18.8 \%$ by $P$. vivax or $P$. ovale, and $4.5 \%$ by $P$. malariae or mixed infections (5). Plasmodium first develops in the liver before emerging up to 1 month later to infect red blood cells. Almost all antimalarials target only the blood stage of the parasite. Therefore, most chemoprophylaxis drugs are taken for 1 month after leaving the malaria area to allow the parasite to reach the targeted blood stage. However, $P$. vivax and $P$. ovale develop hypnozoites, a dormant stage of the parasite in the liver that can emerge months later to cause disease relapses. Treatment of these species requires antirelapse therapy (also known as radical treatment or radical cure). For travelers with intense or prolonged exposure to relapsing species of malaria, presumptive antirelapse therapy (PART) is recommended to kill hypnozoites (๑). Until recently, only primaquine was used for this indication.
Tafenoquine, an 8-aminoquinoline drug related to primaquine, is only the second drug of its class to receive FDA approval. Tafenoquine kills both the liver and blood stages of the parasite, broadening its applicability for chemoprophylaxis to all species of malaria. FDA approved tafenoquine for prophylaxis of malaria in adults aged $\geq 18$ years (Arakoda, $100 \mathrm{mg}$ tablets) in August 2018 and antirelapse therapy of P. vivax malaria in persons aged $\geq 16$ years (Krintafel, $150 \mathrm{mg}$ tablets) in July $2018(7,8)$. Like primaquine, tafenoquine can cause severe hemolysis in persons with glucose-6-phosphate dehydrogenase (G6PD) deficiency and quantitative G6PD testing is required before prescribing. Tafenoquine use is contraindicated in persons with G6PD deficiency (9).

This report summarizes the published efficacy and safety evidence for the recommended doses for both indications and provides guidance for the use of tafenoquine in the United States. A more comprehensive review of the literature on tafenoquine along with the biologic rationale for its use has been published elsewhere (10).

\section{Methods}

CDC conducted a search of English-language articles available in PubMed, Ovid Medline, Scopus, and Global Health (CABDirect) on January 17, 2019, using keywords "tafenoquine or WR238605" and "prevent, prevention, prophylaxis, treatment, radical, or cure." A total of 269 articles were collated and underwent title, abstract, and full text reviews by two physicians: an infectious disease specialist and a malaria subject matter expert. The references from review articles and meta-analyses, and the FDA labeling for both Krintafel and Arakoda were also reviewed to identify any additional studies. Randomized, double-blind, controlled trials performed in human subjects using the labeled recommended dosing regimens were preferentially included in the final review. Data on dosing regimen, outcome, and adverse events were abstracted. Based on the results of the review, CDC subject matter experts developed guidance for the use of tafenoquine.

\section{Rationale and Evidence}

A total of 269 articles were identified. After excluding 232 during title review and 29 during abstract review, the eight remaining articles were reviewed fully and included in the analysis: five articles related to prophylaxis (11-15) and 
three to antirelapse (16-18); among these eight articles, seven included additional information for safety $(11-13,15-18)$. All five studies cited by the FDA label were captured. An additional four peer-reviewed articles addressing the in vivo activity of hypnozoites of $P$. ovale and use of primaquine for $P$. ovale were reviewed $(6,19-21)$; however, because they did not assess tafenoquine use, they were not included in the tafenoquine review.

Prophylaxis. Three of the five articles included were randomized controlled trials (RCTs), one was a reanalysis of data from an RCT, and one was a randomized human challenge study (11-15) (Table 1). Two RCTs compared tafenoquine (200 $\mathrm{mg}$ for 3 days, then weekly thereafter for up to 6 months) to placebo; both found a protective efficacy of $86 \%$ ( $95 \%$ confidence intervals $[\mathrm{CIs}]=73-93$ and 76-92) $(11,12)$. Although not powered to detect statistical differences in efficacy, one of these RCTs described the efficacy between tafenoquine (protective efficacy $=86 \%, 95 \% \mathrm{CI}=76-92$ ) and mefloquine (protective efficacy $=86 \%, 95 \% \mathrm{CI}=72-93)(12)$. The third RCT compared tafenoquine to mefloquine and observed no cases of malaria in either arm (13). The data from this study were reanalyzed in a separate study, using an estimation of attack rate, and found protective efficacy to be $100 \%$ (95\% CI $=93-100)(14)$. These studies suggest comparable efficacy between tafenoquine and mefloquine, the current standard of care. Finally, the randomized human challenge study demonstrated $100 \%$ efficacy $(95 \%$ CI $=40-100)$ of tafenoquine against the blood stage of $P$. falciparum in healthy volunteers compared with placebo (15).

Antirelapse therapy. One phase $2 \mathrm{~b}$ randomized doseselection trial and two phase 3 RCTs examined the efficacy of tafenoquine in the prevention of relapse in patients with confirmed $P$. vivax malaria at the labeled recommended regimen (Table 1) (16-18). Among these studies, tafenoquine was found to prevent relapse in $62 \%-89 \%$ of cases with a single $300 \mathrm{mg}$ dose. In the large phase 2 dose-response study, efficacy of $300 \mathrm{mg}$ and $600 \mathrm{mg}$ were similar, and significantly higher than that of chloroquine alone $(300 \mathrm{mg}$ dose: $89.2 \%$, $95 \% \mathrm{CI}=77-95, \log$-rank test $\mathrm{p}$-value $<0.001 ; 600 \mathrm{mg}$ dose: 91.9\%, 95\% CI $=80-97, \mathrm{p}<0.001$; chloroquine: $36.5 \%$, $95 \% \mathrm{CI}=23-52)(16)$.

P. ovale efficacy. Tafenoquine is not labeled for use in $P$. ovale. Because $P$. ovale is relatively rare, accounting for fewer than $5 \%$ of malaria cases globally (19), it was not evaluated in the tafenoquine studies. Based on the biologic similarity of the hypnozoites of ovale and vivax, a CDC expert committee previously recommended the use of primaquine off-label for antirelapse therapy of $P$. ovale ( 6 ). With similar in vivo response of $P$. ovale to primaquine to that of $P$. vivax $(20,21)$, CDC subject matter experts are extrapolating the use of tafenoquine to P. ovale.

Safety. Seven of the eight reviewed studies provided safety outcomes; four reported safety outcomes at the prophylaxis dose and three at the antirelapse therapy dose (Table 2) (1113,15-18). Common adverse events included abdominal pain, constipation, diarrhea, vertigo, dizziness, sleep disturbances, and headache. Two studies described a nonsignificant increase in methemoglobin $(11,13)$. Another reported asymptomatic decreases in hemoglobin, which resolved without intervention (18). One study described vortex keratopathy (a condition characterized by changes in the corneal epithelium resulting in a whorl pattern) in approximately $90 \%$ of patients receiving tafenoquine prophylaxis; the condition did not affect visual acuity and resolved within 1 year following drug discontinuation (13). Of note, persons with G6PD deficiency were excluded because 8 -aminoquinolines can cause hemolytic anemia in these persons.

\section{Recommendations}

Tafenoquine is an additional FDA-approved antimalarial option for malaria prophylaxis in adults aged $\geq 18$ years, and for antirelapse therapy in persons aged $\geq 16$ years (Box).

Dosage and indication. In adults traveling to areas with malaria, tafenoquine (Arakoda, $100 \mathrm{mg}$ tablets) can be used for chemoprophylaxis for all species of malaria. The prophylactic dose is $200 \mathrm{mg}$ daily for the 3 days preceding the trip, $200 \mathrm{mg}$ weekly during the trip, and a single $200 \mathrm{mg}$ dose during the week after returning. In persons aged $\geq 16$ years, tafenoquine (Krintafel, $150 \mathrm{mg}$ tablets) can be used for presumptive antirelapse therapy or PART for $P$. vivax and off-label for $P$. ovale. The single $300 \mathrm{mg}$ antirelapse or PART dose should ideally overlap with blood-stage treatment or the last dose of prophylaxis. If this is not feasible, tafenoquine may be taken as soon as possible afterwards. PART is not necessary if primaquine or tafenoquine is taken for primary prophylaxis. Tafenoquine should be administered with food.

Contraindications and warnings. Like primaquine, tafenoquine is contraindicated in persons with G6PD deficiency because it might cause hemolytic anemia. If G6PD status is unknown, quantitative G6PD testing must be performed to confirm normal activity before administration of tafenoquine. Qualitative G6PD testing might miss persons with intermediate deficiency and is inadequate to guide tafenoquine administration. Tafenoquine is contraindicated in pregnancy because of the unknown G6PD status of the fetus and should not be used in breastfeeding women if the infant has G6PD deficiency or if the infant's G6PD status is unknown. Because psychiatric adverse reactions were observed in persons with a previous history of psychiatric conditions, tafenoquine should not be used in these 
TABLE 1. Findings from seven blinded, randomized trials of tafenoquine for prophylaxis and antirelapse treatment of malaria at recommended doses

\begin{tabular}{|c|c|c|c|c|c|c|c|c|}
\hline \multirow[b]{2}{*}{ Indication } & \multirow[b]{2}{*}{$\begin{array}{c}\text { Year } \\
\text { published }\end{array}$} & \multirow{2}{*}{$\begin{array}{l}\text { Country } \\
\text { (Plasmodium } \\
\text { species) }\end{array}$} & \multirow[b]{2}{*}{$\begin{array}{l}\text { Study population } \\
\text { characteristics }\end{array}$} & \multirow[b]{2}{*}{ Study length } & \multirow[b]{2}{*}{ Drug regimen } & \multirow[b]{2}{*}{$\begin{array}{c}\text { Sample } \\
\text { size }\end{array}$} & \multicolumn{2}{|c|}{ Treatment } \\
\hline & & & & & & & Outcome & $\begin{array}{l}\% \text { with outcome } \\
\qquad(95 \% \mathrm{Cl})\end{array}$ \\
\hline \multirow[t]{8}{*}{ Prophylaxis } & \multirow[t]{2}{*}{$2001^{*}$} & \multirow{2}{*}{$\begin{array}{l}\text { Kenya } \\
\text { (P. falciparum } \\
\text { primarily) }\end{array}$} & \multirow[t]{2}{*}{ Semi-immune } & \multirow[t]{2}{*}{$\begin{array}{l}13 \text { wks intervention, } \\
\text { follow-up }\end{array}$} & $\begin{array}{l}\text { TQ } 200 \mathrm{mg} \times 3 \text { days, } \\
\text { then weekly }\end{array}$ & 53 & \multirow[t]{2}{*}{ Protective efficacy } & $86(73-93)$ \\
\hline & & & & & Placebo & 59 & & Reference \\
\hline & \multirow[t]{2}{*}{$2003^{\dagger}$} & \multirow{2}{*}{$\begin{array}{l}\text { Ghana } \\
\text { (P. falciparum } \\
\text { primarily) }\end{array}$} & \multirow[t]{2}{*}{ Semi-immune } & \multirow{2}{*}{$\begin{array}{l}12 \text { wks intervention, } \\
4 \text { wks additional } \\
\text { follow-up } \\
\text { (double-blind) }\end{array}$} & $\begin{array}{l}\text { TQ } 200 \mathrm{mg} \times 3 \text { days, } \\
\text { then weekly }\end{array}$ & 91 & \multirow[t]{2}{*}{ Protective efficacy } & $86(76-92)^{\S}$ \\
\hline & & & & & MQ $250 \mathrm{mg} / \mathrm{wk}$ & $\begin{array}{l}46 \\
94\end{array}$ & & $\begin{array}{l}86(72-93)^{\S} \\
\text { Reference }\end{array}$ \\
\hline & \multirow[t]{2}{*}{$2010^{\pi}$} & \multirow[t]{2}{*}{$\begin{array}{l}\text { Timor-Leste } \\
(P . \text { falciparum and } P \text {. } \\
\text { vivax })\end{array}$} & \multirow[t]{2}{*}{ Nonimmune } & \multirow[t]{2}{*}{$\begin{array}{l}6 \text { mos intervention, } \\
\text { follow-up } 20 \text { weeks }\end{array}$} & $\begin{array}{l}\text { TQ } 200 \mathrm{mg} \times 3 \text { days, } \\
\text { then weekly }\end{array}$ & 492 & \multirow[t]{2}{*}{$\begin{array}{l}\text { No. of cases } \\
\text { (protective } \\
\text { efficacy)** }\end{array}$} & $\begin{array}{l}\text { During intervention: } \\
0 \text { cases; During } \\
\text { follow-up: } 4 \text { cases } \\
{[100 \%(93-100)]^{\dagger+}}\end{array}$ \\
\hline & & & & & MQ $250 \mathrm{mg} / \mathrm{wk}$ & 162 & & $\begin{array}{l}\text { During intervention: } 0 ; \\
\text { during follow-up: } \\
1 \text { case [100\% (79-100)] }\end{array}$ \\
\hline & \multirow[t]{2}{*}{$2018^{\S \S}$} & \multirow{2}{*}{$\begin{array}{l}\text { Australia } \\
\text { (P. falciparum } \\
\text { challenge) }\end{array}$} & \multirow[t]{2}{*}{ Healthy, nonimmune } & \multirow[t]{2}{*}{34 days } & $\begin{array}{l}\text { TQ } 200 \mathrm{mg} \times 3 \text { days, } \\
\text { and } 200 \mathrm{mg} \text { on day } 10\end{array}$ & 12 & \multirow[t]{2}{*}{$\begin{array}{l}\text { Rescue treatment } \\
\text { needed }\end{array}$} & $0(0-27)^{9 \uparrow}$ \\
\hline & & & & & Placebo & 4 & & $100(40-100)$ \\
\hline \multirow[t]{8}{*}{$\begin{array}{l}\text { Antirelapse } \\
\text { therapy }\end{array}$} & \multirow[t]{3}{*}{$2014^{* * *}$} & \multirow[t]{3}{*}{$\begin{array}{l}\text { Peru, India, Thailand, } \\
\text { Brazil }\end{array}$} & \multirow{3}{*}{$\begin{array}{l}\geq 16 \text { yrs; microscopically } \\
\text { confirmed } P \text {. vivax } \\
\text { monoinfection }\end{array}$} & \multirow{3}{*}{$\begin{array}{l}180 \text { days from } \\
\text { chloroquine } \\
\text { initiation }\end{array}$} & $\begin{array}{l}\mathrm{CQ} \times 3 \text { days }+\mathrm{TQ} 300 \mathrm{mg} \\
\times 1\end{array}$ & 57 & \multirow{3}{*}{$\begin{array}{l}\text { Relapse-free } \\
\text { efficacy (ITT } \\
\text { population) }\end{array}$} & $89(77-95)^{\dagger+t}$ \\
\hline & & & & & $\begin{array}{l}\mathrm{CQ} \times 3 \text { days }+\mathrm{PQ} 15 \mathrm{mg} \\
\times 14 \text { days }\end{array}$ & 50 & & $77(63-87)^{\dagger+t}$ \\
\hline & & & & & $\mathrm{CQ} \times 3$ days only & 54 & & $38(23-52)$ \\
\hline & \multirow[t]{2}{*}{$2019^{\S \S \S}$} & $\begin{array}{l}\text { Peru, Brazil, } \\
\text { Colombia, Vietnam, }\end{array}$ & $\begin{array}{l}\geq 16 \text { yrs; Hospitalized with } \\
\text { microscopically confirmed }\end{array}$ & 180 days & $\begin{array}{l}\mathrm{CQ} \times 3 \text { days }+\mathrm{TQ} 300 \mathrm{mg} \\
\times 1\end{array}$ & 166 & $\begin{array}{l}\text { Recurrence-free } \\
\text { efficacy (ITT }\end{array}$ & $73(65-79)$ \\
\hline & & Thailand & P. vivax infection & & $\begin{array}{l}\mathrm{CQ} \times 3 \text { days }+\mathrm{PQ} 15 \mathrm{mg} / \\
\text { day } \times 14 \text { days }\end{array}$ & 85 & population) & $75(64-83)$ \\
\hline & 2019 ตางๆ & $\begin{array}{l}\text { Peru, Brazil, Ethiopia, } \\
\text { Cambodia, Thailand, }\end{array}$ & $\begin{array}{l}\geq 16 \text { yrs ( } \geq 18 \text { in Ethiopia); } \\
\text { microscopically confirmed }\end{array}$ & 180 days & $\begin{array}{l}\mathrm{CQ} \times 3 \text { days }+\mathrm{TQ} 300 \mathrm{mg} \\
\times 1\end{array}$ & 260 & $\begin{array}{l}\text { Recurrence-free } \\
\text { efficacy (ITT }\end{array}$ & $62(55-69)^{* * * *}$ \\
\hline & & Philippines & P. vivax infection & & $\begin{array}{l}\mathrm{CQ} \times 3 \text { days }+\mathrm{PQ} 15 \mathrm{mg} / \\
\text { day } \times 14 \text { days }\end{array}$ & 133 & population) & $70(60-77)$ \\
\hline & & & & & Placebo & 129 & & $28(20-36)$ \\
\hline
\end{tabular}

Abbreviations: $\mathrm{Cl}=$ confidence interval; $\mathrm{CQ}=$ chloroquine; $\mathrm{ITT}=$ intention to treat; $\mathrm{MQ}=$ mefloquine; $\mathrm{PQ}=$ primaquine; $\mathrm{TQ}=$ tafenoquine.

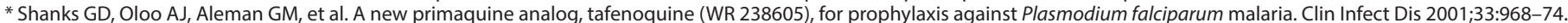

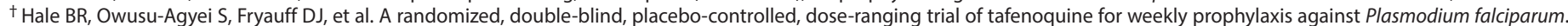

Clin Infect Dis 2003;36:541-9.

$\S$ Chi squared test $(p<0.05)$.

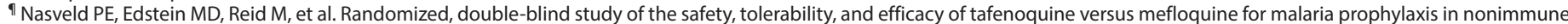
subjects. Antimicrob Agents Chemother 2010;54:792-8.

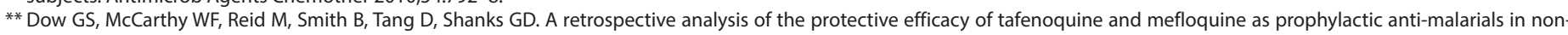
immune individuals during deployment to an area with endemic malaria area. Malar J 2014;13:49.

†† Fisher exact TQ versus $\mathrm{MQ} p=1.0$

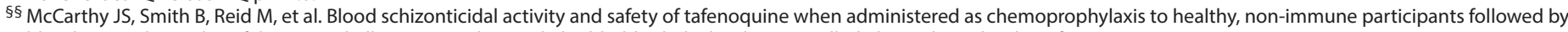
blood stage Plasmodium falciparum challenge: a randomized, double-blinded, placebo-controlled Phase 1b study. Clin Infect Dis 2019;69:480-6.

१ी Fisher exact $\mathrm{p}<0.005$.

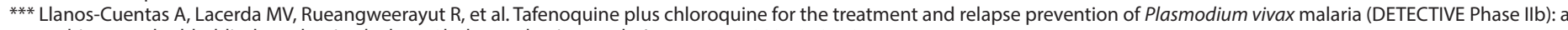
multicentre, double-blind, randomized, phase 2 b dose-selection study. Lancet 2014;383:1049-58.

${ }^{+\dagger+}$ Log-rank TQ versus placebo $p<0.0001 ; P Q$ versus placebo $p=0.0004$.

$\S \S \S$ Llanos-Cuentas A, Lacerda MVG, Hien TT, et al. Tafenoquine versus primaquine to prevent relapse of Plasmodium vivax malaria (GATHER). N Engl J Med 2019;380:229-41.

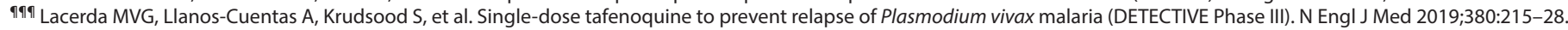
**** TQ hazard ratio (HR) 0.3; PQ HR 0.26, p<0.001.

persons $(3,4)$, and other antimalarials could be considered for prophylaxis, or primaquine can be considered as an alternative for antirelapse therapy. Tafenoquine is contraindicated in persons with known hypersensitivity to 8-aminoquinolines.

Adverse events and reporting. Adverse events might be delayed in onset or duration because of tafenoquine's long half-life. Common adverse reactions include dizziness, nausea, vomiting, and headache. When used for prophylaxis, elevated liver enzyme levels, insomnia, depression, abnormal dreams, and anxiety were also observed. Suspected adverse reactions can be reported to FDA via MedWatch at https://www.fda. gov/safety/medwatch.

\section{Discussion}

This guidance regarding use of tafenoquine for both prophylaxis of all species of malaria and antirelapse therapy for P. vivax is consistent with FDA labeling. Recommendations for PART and antirelapse therapy of $P$. ovale are off-label. It is not feasible to conduct adequately powered clinical trials for $P$. ovale malaria because of its relatively low incidence. Therefore, evidence for efficacy against $P$. vivax was extrapolated to $P$. ovale.

For persons with contraindications to tafenoquine, other antimalarial options for malaria chemoprophylaxis and radical cure can be considered. There are several other options for chemoprophylaxis, each with its own contraindications and 
TABLE 2. Summary of key adverse events observed in persons receiving tafenoquine at recommended doses versus placebo or mefloquine

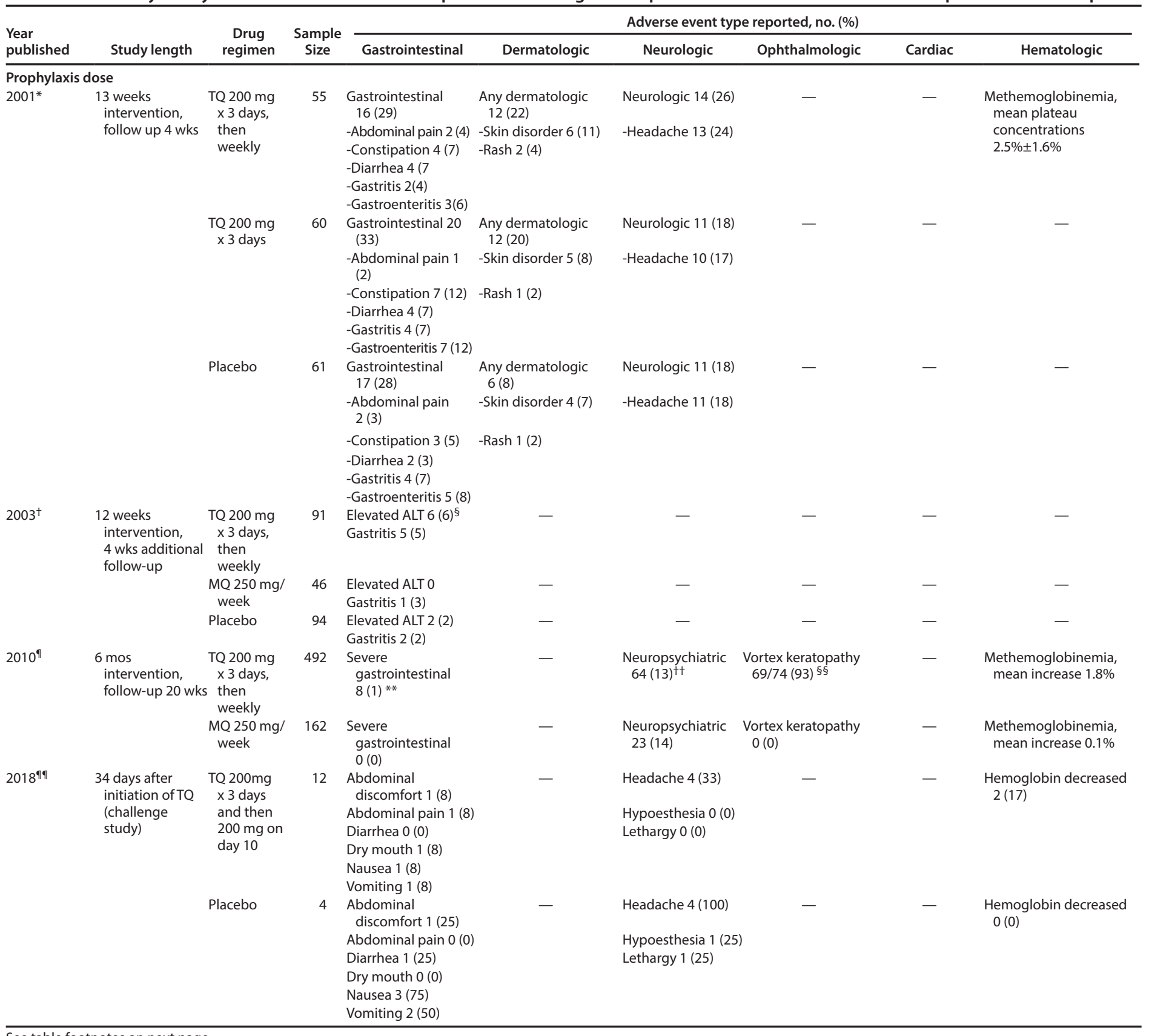

warnings, which can be used depending on the patient and drug-resistance in the areas of travel. These include atovaquoneproguanil, chloroquine, doxycycline, and mefloquine. For antirelapse therapy, the only alternative is primaquine. For nonpregnant persons with borderline or intermediate G6PD deficiency requiring antirelapse treatment, an alternative dosing regimen of primaquine could be considered at $45 \mathrm{mg}$ (base) once weekly for 8 weeks, with close monitoring and consultation with an infectious disease expert. Persons with severe G6PD deficiency will require antimalarials at prophylaxis doses for 1 year instead of an 8-aminoquinoline (i.e., primaquine or tafenoquine). Pregnant women with normal G6PD levels, requiring antirelapse therapy could be given chloroquine at chemoprophylaxis doses (500 mg salt once weekly) until after delivery, and then an 8-aminoquinoline, depending on whether the woman is breastfeeding and the G6PD status of the infant.

The approval of tafenoquine marks a notable advancement for the prevention of malaria and treatment of $P$. vivax and $P$. ovale. Its long half-life of 15 days allows for weekly prophylactic dosing during travel and a single dose for antirelapse 
TABLE 2. (Continued) Summary of key adverse events observed in persons receiving tafenoquine at recommended doses versus placebo or mefloquine

\begin{tabular}{|c|c|c|c|c|c|c|c|c|c|}
\hline \multirow{2}{*}{$\begin{array}{l}\text { Year } \\
\text { published }\end{array}$} & \multirow[b]{2}{*}{ Study length } & \multirow{2}{*}{$\begin{array}{l}\text { Drug } \\
\text { regimen }\end{array}$} & \multirow{2}{*}{$\begin{array}{l}\text { Sample } \\
\text { Size }\end{array}$} & \multicolumn{6}{|c|}{ Adverse event type reported, no. (\%) } \\
\hline & & & & Gastrointestinal & Dermatologic & Neurologic & Ophthalmologic & Cardiac & Hematologic \\
\hline \multicolumn{10}{|c|}{ Antirelapse therapy dose } \\
\hline \multirow[t]{4}{*}{$2014 * * *$} & \multirow[t]{4}{*}{$\begin{array}{l}\text { Follow up to } \\
180 \text { days } \\
\text { posttreatment }\end{array}$} & $\begin{array}{l}\text { TQ } 300 \mathrm{mg} \\
\text { plus CQ }\end{array}$ & 57 & $\begin{array}{l}\text { Upper abdominal } \\
\text { pain } 6(11) \\
\text { Nausea } 5(9)\end{array}$ & Pruritus 8 (14) & $\begin{array}{l}\text { Asthenia } 5 \text { (9) } \\
\text { Insomnia } 5 \text { (9) }\end{array}$ & - & $\begin{array}{l}\text { QT prolongation } \\
3(5)\end{array}$ & Anemia 1 (2) \\
\hline & & $\begin{array}{l}\text { PQ } 15 \mathrm{mg} \\
\text { plus CQ }\end{array}$ & 50 & $\begin{array}{l}\text { Upper abdominal } \\
\text { pain } 7(14) \\
\text { Nausea } 4(8)\end{array}$ & Pruritus 3 (6) & $\begin{array}{l}\text { Asthenia } 0(0) \\
\text { Insomnia } 3(6)\end{array}$ & - & $\begin{array}{l}\text { QT prolongation } \\
5(10)\end{array}$ & Anemia $0(0)$ \\
\hline & & \multirow[t]{2}{*}{ CQ only } & \multirow[t]{2}{*}{54} & $\begin{array}{l}\text { Upper abdominal } \\
\text { pain } 5(9)\end{array}$ & \multirow[t]{2}{*}{ Pruritus 7 (13) } & Asthenia $0(0)$ & - & \multirow[t]{2}{*}{$\begin{array}{l}\text { QT prolongation } \\
4 \text { (7) }\end{array}$} & \multirow[t]{2}{*}{ Anemia $0(0)$} \\
\hline & & & & Nausea $3(6)$ & & Insomnia 1 (2) & & & \\
\hline \multirow[t]{4}{*}{$2019^{t+\dagger}$} & \multirow{4}{*}{$\begin{array}{l}\text { Follow up to } \\
180 \text { days } \\
\text { posttreatment }\end{array}$} & \multirow[t]{2}{*}{$\begin{array}{l}\text { TQ } 300 \mathrm{mg} \\
\text { plus CQ }\end{array}$} & \multirow[t]{2}{*}{166} & Nausea $16(10)$ & \multirow[t]{2}{*}{ Pruritus 20 (12) } & Dizziness 27 (16) & $\begin{array}{l}\text { Vortex keratopathy } \\
1 \text { (1) }\end{array}$ & \multirow[t]{2}{*}{ - } & \multirow[t]{2}{*}{ - } \\
\hline & & & & Vomiting 11 (7) & & Headache 19 (11) & $\begin{array}{l}\text { Retinal hypo- } \\
\text { pigmentation } 1(1) \\
\text { Retinal hyper- } \\
\text { pigmentation } 1(1)\end{array}$ & & \\
\hline & & \multirow[t]{2}{*}{$\begin{array}{l}\mathrm{PQ} 15 \mathrm{mg} \\
\text { plus CQ }\end{array}$} & \multirow[t]{2}{*}{85} & Nausea 6 (7) & \multirow[t]{2}{*}{ Pruritus 19 (22) } & Dizziness 13 (15) & $\begin{array}{l}\text { Retinal hypo- } \\
\text { pigmentation } 1 \text { (2) }\end{array}$ & \multirow[t]{2}{*}{ - } & - \\
\hline & & & & Vomiting 5 (6) & & Headache $10(12)$ & & & \\
\hline $2019 \S \S \S$ & $\begin{array}{l}\text { Follow up to } \\
180 \text { days }\end{array}$ & $\begin{array}{l}\text { TQ } 300 \mathrm{mg} \\
\text { plus CQ }\end{array}$ & 260 & Nausea $16(6)$ & Pruritus 127 (49) & Dizziness 22 (9) & $\begin{array}{l}\text { Unilateral } \\
\text { keratopathy } 1\end{array}$ & - & $\begin{array}{l}\text { Hemoglobin decreased } \\
>3 \mathrm{~g} / \mathrm{dL} 14 \text { (5) }\end{array}$ \\
\hline & posttreatment & & & Vomiting 15 (6) & & Headache 12 (5) & Unilateral retinal & & \\
\hline & & & & $\begin{array}{l}\text { Diarrhea } 10(4) \\
\text { Upper abdominal } \\
\text { pain } 8(3) \\
\text { Elevated ALT } 6(2)\end{array}$ & & & change 2 & & \\
\hline & & $\begin{array}{l}\mathrm{PQ} 15 \mathrm{mg} \\
\text { plus } \mathrm{CQ}\end{array}$ & 129 & $\begin{array}{l}\text { Nausea } 7(5) \\
\text { Vomiting } 9(7)\end{array}$ & Pruritus 14 (11) & $\begin{array}{l}\text { Dizziness } 8(6) \\
\text { Headache } 5(4)\end{array}$ & $\begin{array}{l}\text { Retinal hypo- } \\
\text { pigmentation } 1\end{array}$ & - & $\begin{array}{l}\text { Hemoglobin decreased } \\
>3 \mathrm{~g} / \mathrm{dL} 2 \text { (2) }\end{array}$ \\
\hline & & & & Diarrhea $2(2)$ & & & & & \\
\hline & & & & $\begin{array}{l}\text { Upper abdominal } \\
\text { pain } 6(5)\end{array}$ & & & & & \\
\hline & & & & Elevated ALT 3 (2) & & & & & \\
\hline & & CQ only & 133 & Nausea $9(7)$ & Pruritus 17 (13) & Dizziness 4 (3) & - & - & Hemoglobin decreased \\
\hline & & & & Vomiting 7 (5) & & Headache 9 (7) & & & $>3 \mathrm{~g} / \mathrm{dL} 2(2)$ \\
\hline & & & & Diarrhea 4 (3) & & & & & \\
\hline & & & & $\begin{array}{l}\text { Upper abdominal } \\
\text { pain } 9(7)\end{array}$ & & & & & \\
\hline & & & & Elevated ALT 6 (5) & & & & & \\
\hline
\end{tabular}

Abbreviations: $\mathrm{ALT}=$ alanine aminotransferase; $\mathrm{CQ}=$ chloroquine; $\mathrm{MQ}=$ mefloquine; $\mathrm{PQ}=$ primaquine; $\mathrm{TQ}=$ tafenoquine .

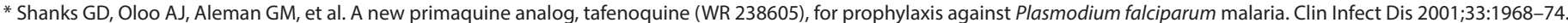

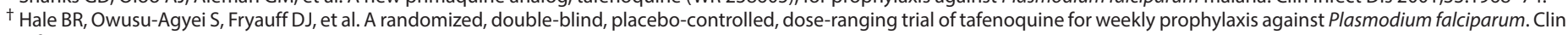
Infect Dis 2003;36:541-9.

$\S$ For all six, ALT exceeded a predetermined threshold and returned to normal levels when drug was discontinued. No clinical significance.

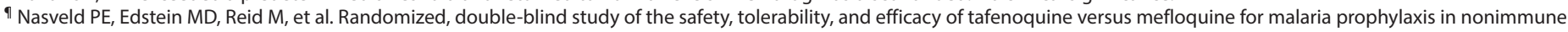
subjects. Antimicrob Agents Chemother 2010;54:792-8.

** Most common gastrointestinal events: abdominal pain, constipation, and diarrhea. No difference between tafenoquine and mefloquine gastrointestinal events.

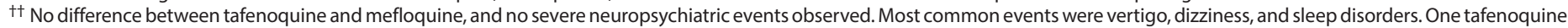
subject withdrew because of depression (moderate), and one for hyperesthesia (moderate).

$\S \S$ Subset analysis for vortex keratopathy. Not associated with visual disturbances and resolved by 1 year.

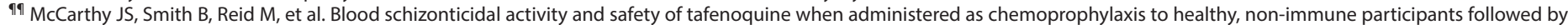
blood stage Plasmodium falciparum challenge: a randomized, double-blinded, placebo-controlled Phase 1b study. Clin Infect Dis 2019;69:480-6.

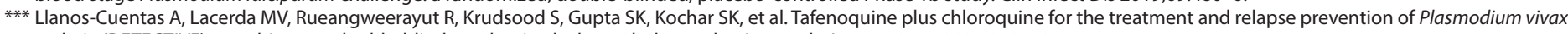
malaria (DETECTIVE): a multicentre, double-blind, randomized, phase 2b dose-selection study. Lancet. 2014;383:1049-58.

†+† Llanos-Cuentas A, Lacerda MVG, Hien TT, et al. Tafenoquine versus primaquine to prevent relapse of Plasmodium vivax malaria (GATHER). N Engl J Med 2019;380:229-41.

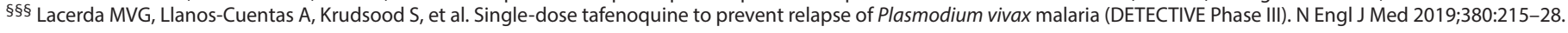

therapy, which has the potential to increase adherence for both indications $(7,8)$. With two strengths of tafenoquine tablets available, it is important that clinicians ensure that the appropriate dose is used for each specific indication.

Malaria is a notifiable disease in the United States. CDC's National Malaria Surveillance System collects information about cases of malaria occurring in the United States, providing an opportunity to assess the use and clinical outcomes of tafenoquine. Postmarketing surveillance is being conducted to monitor the occurrence of adverse events. Adverse events related to tafenoquine should be reported voluntarily to FDA's MedWatch adverse event reporting system, and as part of routine reporting to CDC.

Corresponding author: Kathrine R. Tan, ktan@cdc.gov, 404-718-4701.

\footnotetext{
${ }^{1}$ Department of Pediatrics, Emory University School of Medicine, Atlanta, Georgia; ${ }^{2}$ Division of Parasitic Diseases and Malaria, Center for Global Health, CDC; ${ }^{3}$ U.S. President's Malaria Initiative, Washington, DC.
} 


\section{Summary}

What is already known about this topic?

Malaria can be prevented by taking antimalarials when traveling to an area with malaria. Treatment of malaria caused by Plasmodium vivax and Plasmodium ovale requires antirelapse therapy to kill the dormant liver-stage parasite.

What is added by this report?

Adults aged $\geq 18$ years can take tafenoquine (Arakoda $100 \mathrm{mg}$ tablets) to prevent malaria. Persons aged $\geq 16$ years requiring antirelapse therapy for $P$. vivax or $P$. ovale can take tafenoquine (Krintafel 150 mg tablets). Before using tafenoquine, quantitative testing to rule out glucose-6-phosphate dehydrogenase deficiency is required.

What are the implications for public health practice?

Tafenoquine is another option for malaria chemoprophylaxis and for antirelapse therapy. The simplified dosing regimen has the potential to improve adherence.

\section{BOX. Guidance for the use of tafenoquine by indication*}

\section{Prophylaxis $^{\dagger}$}

- Loading regimen

- 200 mg daily by mouth x 3 days before departure

- Maintenance regimen

- $200 \mathrm{mg}$ once weekly by mouth beginning 7 days after last loading dose

- Continue for entire duration of travel plus one additional dose after returning

- Take on the same day of the week each week

\section{Antirelapse therapy $\$$}

- Single $300 \mathrm{mg}$ dose by mouth, ideally on the first or second day of blood-stage treatment

- If not feasible to overlap with blood-stage treatment, may be taken as soon as possible afterwards

\section{Presumptive antirelapse therapy ${ }^{\S}$}

- Single $300 \mathrm{mg}$ dose by mouth, ideally on the same day as the last dose of prophylaxis

- If not feasible to overlap with last dose of prophylaxis, may be taken as soon as possible afterwards

- Antirelapse dose not needed if primaquine or tafenoquine is used for prophylaxis

\footnotetext{
*Contraindications: glucose-6-phosphate dehydrogenase (G6PD) deficiency, pregnancy, breastfeeding (if infant has G6PD deficiency or if G6PD status is unknown), known hypersensitivity to 8-aminoquinolines, history of psychiatric disorder.

$\dagger$ Persons aged $\geq 18$ years.

$\$$ Persons aged $\geq 16$ years.
}

All authors have completed and submitted the International Committee of Medical Journal Editors form for disclosure of potential conflicts of interest. Kathrine Tan reports that she is a coinvestigator for postmarketing surveillance for adverse events associated with tafenoquine use; she receives no compensation for this work. No other potential conflicts of interest were disclosed.

\section{References}

1. World Health Organization. World malaria report 2018. Geneva, Switzerland: World Health Organization; 2018. https://www.who.int/ malaria/publications/world-malaria-report-2018/en/

2. CDC. Yellow book 2018: health information for international travel. New York, NY: Oxford University Press; 2017.

3. Food and Drug Administration. Krintafel (tafenoquine) tablets. GlaxoSmithKline. Silver Spring, MD: US Department of Health and Human Services, Food and Drug Administration; 2018. https://www. accessdata.fda.gov/drugsatfda_docs/nda/2018/210795Orig1s000TOC.cfm

4. Food and Drug Administration. Arakoda (tafenoquine) tablets. Sixty Degrees Pharmaceuticals, LLC. Silver Spring, MD: US Department of Health and Human Services, Food and Drug Administration; 2018. https://www.accessdata.fda.gov/drugsatfda_docs/ nda/2018/210607Orig1s000TOC.cfm

5. Mace KE, Arguin PM, Lucchi NW, Tan KR. Malaria surveillanceUnited States, 2016. MMWR Surveill Summ 2019;68(No. SS-5).

6. Hill DR, Baird JK, Parise ME, Lewis LS, Ryan ET, Magill AJ. Primaquine: report from $\mathrm{CDC}$ expert meeting on malaria chemoprophylaxis I. Am J Trop Med Hyg 2006;75:402-15. https://doi.org/10.4269/ ajtmh.2006.75.402

7. Food and Drug Administration. Arakoda (tafenoquine) tablets. Sixty Degrees Pharmaceuticals, LLC. Silver Spring, MD: US Department of Health and Human Services, Food and Drug Administration; 2018. https://www. accessdata.fda.gov/drugsatfda_docs/label/2018/210607lbl.pdf

8. Food and Drug Administration. Krintafel (tafenoquine) tablets. GlaxoSmithKline. Silver Spring, MD: US Department of Health and Human Services, Food and Drug Administration; 2018. https://www. accessdata.fda.gov/drugsatfda_docs/label/2018/210795s000lbl.pdf

9. Rueangweerayut R, Bancone G, Harrell EJ, et al. Hemolytic potential of tafenoquine in female volunteers heterozygous for glucose-6-phosphate dehydrogenase (G6PD) deficiency (G6PD Mahidol Variant) versus G6PD-normal volunteers. Am J Trop Med Hyg 2017;97:702-11. https://doi.org/10.4269/ajtmh.16-0779

10. Baird JK. Tafenoquine for travelers' malaria: evidence, rationale and recommendations. JTravel Med 2018;25. https://doi.org/10.1093/jtm/tay110

11. Shanks GD, Oloo AJ, Aleman GM, et al. A new primaquine analogue, tafenoquine (WR 238605), for prophylaxis against Plasmodium falciparum malaria. Clin Infect Dis 2001;33:1968-74. https://doi. org/10.1086/324081

12. Hale BR, Owusu-Agyei S, Fryauff DJ, et al. A randomized, doubleblind, placebo-controlled, dose-ranging trial of tafenoquine for weekly prophylaxis against Plasmodium falciparum. Clin Infect Dis 2003;36:541-9. https://doi.org/10.1086/367542

13. Nasveld PE, Edstein MD, Reid M, et al.; Tafenoquine Study Team. Randomized, double-blind study of the safety, tolerability, and efficacy of tafenoquine versus mefloquine for malaria prophylaxis in nonimmune subjects. Antimicrob Agents Chemother 2010;54:792-8. https://doi. org/10.1128/AAC.00354-09

14. Dow GS, McCarthy WF, Reid M, Smith B, Tang D, Shanks GD. A retrospective analysis of the protective efficacy of tafenoquine and mefloquine as prophylactic anti-malarials in non-immune individuals during deployment to a malaria-endemic area. Malar J 2014;13:49. https://doi.org/10.1186/1475-2875-13-49 
15. McCarthy JS, Smith B, Reid M, et al. Blood schizonticidal activity and safety of tafenoquine when administered as chemoprophylaxis to healthy, non-immune participants followed by blood stage Plasmodium falciparum challenge: a randomized, double-blinded, placebo-controlled Phase 1b study. Clin Infect Dis 2018;69:480-6. https://doi.org/10.1093/ $\mathrm{cid} /$ ciy939

16. Llanos-Cuentas A, Lacerda MV, Rueangweerayut R, et al. Tafenoquine plus chloroquine for the treatment and relapse prevention of Plasmodium vivax malaria (DETECTIVE): a multicentre, double-blind, randomised, phase 2b dose-selection study. Lancet 2014;383:1049-58. https://doi. org/10.1016/S0140-6736(13)62568-4

17. Llanos-Cuentas A, Lacerda MVG, Hien TT, et al. Tafenoquine versus primaquine to prevent relapse of Plasmodium vivax malaria. N Engl J Med 2019;380:229-41. https://doi.org/10.1056/NEJMoa1802537
18. Lacerda MVG, Llanos-Cuentas A, Krudsood S, et al. Singledose tafenoquine to prevent relapse of Plasmodium vivax malaria (DETECTIVE Phase III). N Engl J Med 2019;380:215-28. https:// doi.org/10.1056/NEJMoa1710775

19. Mueller I, Zimmerman PA, Reeder JC. Plasmodium malariae and Plasmodium ovale - the "bashful" malaria parasites. Trends Parasitol 2007;23:278-83. https://doi.org/10.1016/j.pt.2007.04.009

20. Shimizu S, Kikuchi T, Koga M, et al.; Research Group on Chemotherapy of Tropical Diseases. Optimal primaquine use for radical cure of Plasmodium vivax and Plasmodium ovale malaria in Japanese travelers-a retrospective analysis. Travel Med Infect Dis 2015;13:235-40. https:// doi.org/10.1016/j.tmaid.2014.11.005

21. Faucher JF, Bellanger AP, Chirouze C, Hustache-Mathieu L, Genton S, Hoen B. Primaquine for radical cure of Plasmodium vivax and Plasmodium ovale malaria: an observational survey (2008-2010). J Travel Med 2013;20:134-6. https://doi.org/10.1111/jtm.12009 